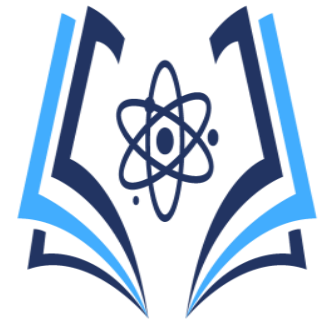

Mercedes Lucas Choez ${ }^{1}$

Martha Quiroz Figueroa ${ }^{2}$

\section{Rol de la enfermera en pacientes con complicaciones postquirúrgicas renales y de las vías urinarias}

\author{
Role of the nurse in patients with postoperative renal and \\ urinary tract complications
}

\section{Rol de la enfermera en pacientes con complicaciones}

${ }^{1}$ Universidad Estatal del Sur de Manabí, mercedes.lucas@ unesum.edu.ec, https://orcid.org/0000-0003-20017611

${ }^{2}$ Universidad Estatal del Sur de Manabí, martha.quiroz@unesum.edu.ec, https://orcid.org/0000-0002-0959-0591

Contacto: mercedes.lucas@unesum.edu.ec

Recibido: 21-09-2019 Aprobado: 15-12-2019

\title{
Resumen
}

El profesional de Enfermería se encarga de valorar al individuo e identificar las necesidades del paciente de orígenes fisiológicos, psicológicos y sociales basados en el conocimiento científico con el fin de establecer o conservar la salud y bienestar del paciente antes, durante y después de un procedimiento quirúrgico. El objetivo de esta investigación fue identificar el rol de la enfermera en pacientes con complicaciones postquirúrgicas renales y de las vías urinarias. A partir de una revisión integrativa de la literatura, se tomaron a consideración diferentes bases de datos de carácter regional e internacional como: Lilacs, Cochrane Library y PubMed. La población estuvo conformada por 120 artículos de los cuales fueron seleccionados 19 artículos en idioma español, inglés y portugués con una antigüedad no mayor a cinco años. En los resultados de esta revisión bibliográfica se pudo comprobar que, 6 artículos hacen referencia al rol asistencial que desempeña la enfermera/o ante las complicaciones postquirúrgicas renales y de las vías urinarias. Las complicaciones postquirúrgicas renales y de las vías urinarias abarcan un factor de complejidad tras las intervenciones quirúrgicas, entre ella la principal complicación es la Insuficiencia Renal Aguda seguido de, Infección del Tracto Urinario, Retención Urinaria y la Estenosis uretral. Esta revisión demuestra que el rol que más se desempeña es el rol asistencial siendo estas intervenciones de enfermería un logro que permite un mejor proceso quirúrgico, ya que tienen un nivel de significancia en el cuidado de este, mejorando la estancia durante la etapa postoperatoria, disminuyendo la estancia hospitalaria.

\begin{abstract}
Palabras claves: Complicaciones posoperatorias, intervención de enfermería, rol asistencial

Abstract

The Nursing professional is in charge of assessing the individual and identifying the needs of the patient of physiological, psychological and social origin based on scientific knowledge in order to establish or preserve the health and wellbeing of the patient before, during and after a surgical procedure. The objective of this research was to identify the role of the nurse in patients with postoperative renal and urinary tract complications. Based on an integrative review of the literature, different regional and international databases were considered, such as: Lilacs, Cochrane Library, and PubMed. The population consisted of 120 articles from which 19 articles in Spanish, English and Portuguese were selected with an antiquity of no more than five years. In the results of this bibliographic review, it was found that 6 articles refer to the care role played by the nurse in the face of postsurgical renal and urinary tract complications. Post-surgical renal and urinary tract complications include a complexity factor after surgical interventions, among which the main complication is Acute Renal Insufficiency followed by Urinary Tract Infection, Urinary Retention and Urethral Stenosis. This review demonstrates that the role that is most played is the care role, these nursing interventions being an achievement that allows a better surgical process, since they have a level of significance in the care of this, improving the stay during the postoperative stage, reducing the hospital stay.
\end{abstract}


Key words: Postoperative complications, nursing intervention, care role.

Introducción

El Rol de la enfermera en los pacientes con complicaciones renales tiene un alto impacto en la etapa postoperatoria ya que son efectos adversos debido a varios factores, entre ellos los propios de cada cirugía como son la localización de la misma, el tipo de intervención o la técnica anestésica utilizada; así como las condiciones del paciente tales como el sexo, la edad y factores socioculturales. Es de vital importancia que el profesional de enfermería preste cuidados centrados en las respuestas humanas, tratando a las personas como un todo; ya que el paciente es un individuo único, que necesita cuidados enfocados no solo en su estado físico sino psicológico.

El rol del profesional de enfermería son un conjunto de acciones para el cuidado de la vida y salud de las personas, promoción de la salud, prevención de enfermedades, esta se basa en 4 funciones, las cuales son, asistencial, educativo, administrativa e investigativa, estas nos ayudan a mejorar el desempeño óptimo de los servicios de salud y además de que permiten al personal desenvolverse en el área que más les guste.

La enfermera profesional requiere una forma de actuar encaminada a solucionar o minimizar los posibles problemas de la vida cotidiana relacionados con la salud, siendo su actuación ordenada y sistematizada, donde las acciones estén estructuradas y direccionadas a la resolución de las necesidades y a potencializar la independencia de quien recibe los cuidados profesionales de enfermería (1).

$\mathrm{La}$ estructura de las actividades se refleja mediante la aplicación del Proceso de Atención de Enfermería (PAE), que es una herramienta para organizar el trabajo del profesional. Mediante la cual se encuentra constituida por una serie de pasos o etapas íntimamente interrelacionadas. Dichas etapas son la valoración, diagnóstico, planificación, ejecución y evaluación (2).

Por otra parte, la fase postoperatoria es el periodo que transcurre entre el final de una operación y la recuperación completa o parcial del paciente con o sin secuelas, a su vez se divide en el en inmediato, mediato y tardío. Las funciones y rol de enfermería en esta etapa son muy importante para la recuperación del paciente (3). Las complicaciones postoperatorias pueden ocurrir como resultado del tratamiento que se realice al paciente, mas no por la patología que padece, el mismo que podría ser evitado en algunos casos, con una buena planificación y ejecución de las cirugías propuestas.

Siendo la insuficiencia renal aguda postoperatoria una de las principales complicaciones, además encontramos la infección del tracto urinario, retención urinaria, la estenosis uretral, trombosis de la vena renal y el cólico renal. Estas complicaciones se suelen dar tras las intervenciones cardiacas, la alteración en la función renal ocurre posterior a la lesión renal en túbulos, vasos, intersticio y glomérulos y excepcionalmente sin una lesión demostrable o puede ser consecuencia de la agudización en un paciente con enfermedad renal previa. La insuficiencia postrenal lleva consigo el concepto de obstrucción de la vía excretora. Esta obstrucción puede producirse en cualquier punto de la vía urinaria, desde la pelvis hasta el meato urinario (4).

Los efectos de las intervenciones quirúrgicas mayores, de la anestesia y del traumatismo estimulan la secreción de la hormona antidiurética y provocan una disminución de la tasa de filtración del flujo sanguíneo renal. La estimulación de la secreción de la hormona antidiurética después de cirugía o de trauma lleva consigo un aumento de la concentración urinaria con disminución del volumen urinario y, en consecuencia, un posible aumento de la insuficiencia renal aguda.

Este proyecto está enfocado en realizar una revisión bibliográfica sobre el rol que desempeña el profesional de enfermería, dejando como ejemplo la elaboración de un plan de cuidados para la aplicación del P.A.E en un paciente con posibles complicaciones postoperatorias renales, con la finalidad que este plan sea de utilidad para las intervenciones correspondientes ante las acciones de enfermería que solucionen el estado de salud del paciente.

\section{Materiales y métodos}

En la presente revisión bibliográfica, se tomaron a consideración diferentes bases de datos de carácter regional e internacional tales como: Lilacs, Cochrane Library, PubMed.

\section{Diseño de estudio}

Las revisiones sistemáticas son investigaciones científicas en las cuales la unidad de análisis son los estudios originales primarios de los cuales se extraen la información pertinente. Además, son indispensables para la práctica de enfermería basada en la evidencia y una herramienta fundamental en la toma de decisiones.

\section{Población y muestra}

La población estuvo conformada por 120 artículos de los cuales fueron seleccionados 19 artículos para la presente revisión bibliográfica difundidos en las bases de datos científicos y que responden a artículos notificados en idioma español, inglés y portugués con una antigüedad no mayor a cinco años. Por otra parte, se excluyeron las revisiones bibliográficas realizadas antes del 2015. Teniendo como referencia las siguientes palabras claves para la búsqueda oportuna. 
$>$ Postoperative complications or Nursing role

$>$ Insuficiencia renal y septicemia

$>$ Complicación postoperatoria renal

$>$ Incidencia, análisis, tracto urinario

$>$ Lesión renal y Trasplante

$>$ Catéter urinario, Complicaciones

$>$ Nefropatía e infección

Técnica de análisis

La técnica utilizada fue la recopilación bibliográfica, mediante el análisis exhaustivo de documentos fiables que presentan la información requerida. El estudio de la revisión sistemática está constituido por la elaboración de una tabla resumen con los datos principales de cada uno de los artículos seleccionados, con la finalidad de obtener el análisis correspondiente y de esta manera se pudo realizar la evidencia aportada por cada revisión a consideración.

Aspectos éticos

La valoración de los artículos científicos investigados, están de acuerdo con las normas técnicas de la bioética en la investigación, evidenciando que cada uno de ellos haya dado cumplimiento a los principios éticos en su realización.

\section{Resultados}

Se presentan los resultados de la revisión bibliográfica concerniente al rol de la en enfermera en complicaciones postoperatorias renales y de las vías urinarias, para lo cual se tomaron diversos estudios publicados desde el 2015 hasta el 2020 en revistas científicas indexadas regionales e internacionales.

\begin{tabular}{|c|c|c|c|c|c|}
\hline $\begin{array}{c}\text { Autores y } \\
\text { nombre de } \\
\text { estudio }\end{array}$ & Revista & $\begin{array}{l}\text { Palabra } \\
\text { Clave }\end{array}$ & Ã̃o & Complicaciones & Rol de enfermería \\
\hline $\begin{array}{l}\text { Bzoma, Kostro, } \\
\text { Hellmann, } \\
\text { Chamienia, } \\
\text { Hać, Ślizień, } \\
\text { Śledziński } \\
\text { Colocación de } \\
\text { Stents ureterales } \\
\text { en receptores de } \\
\text { trasplantes de } \\
\text { riñón, } \\
\text { experiencia del } \\
\text { centro } \\
\text { Gdansk, Polonia } \\
\text { (25) }\end{array}$ & PubMed & $\begin{array}{l}\text { Postoperativ } \\
\text { e } \\
\text { complication } \\
\text { s, Nursing } \\
\text { role }\end{array}$ & 2018 & $\begin{array}{l}\text { Pérdidas de orina y } \\
\text { estenosis del uréter, } \\
\text { pero también puede } \\
\text { asociarse con } \\
\text { complicaciones } \\
\text { específicas, en } \\
\text { particular } \\
\text { infecciones del } \\
\text { tracto urinario. }\end{array}$ & $\begin{array}{l}\text { No se aplicó ningún } \\
\text { rol de enfermería }\end{array}$ \\
\hline 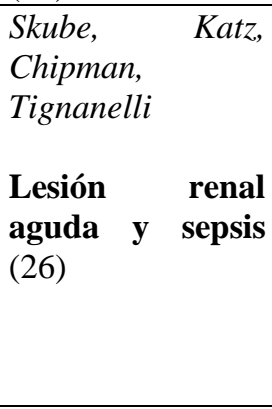 & PubMed & $\begin{array}{l}\text { Insuficiencia } \\
; \\
\text { Renal; septic } \\
\text { emia. }\end{array}$ & 2018 & $\begin{array}{l}\text { La insuficiencia } \\
\text { renal aguda (IRA) } \\
\text { como la sepsis son } \\
\text { complicaciones } \\
\text { clínicas } \\
\text { importantes. La } \\
\text { sepsis es una causa } \\
\text { importante y es una } \\
\text { complicación } \\
\text { común. }\end{array}$ & $\begin{array}{l}\text { No se aplicó ningún } \\
\text { rol de enfermería }\end{array}$ \\
\hline $\begin{array}{l}\text { Krajka, Dorobek } \\
\text { Impacto de la } \\
\text { sonda vesical } \\
\text { urinaria tras la } \\
\text { extracción de } \\
\text { cálculos } \\
\text { ureterorrenoscó } \\
\text { picos en el } \\
\text { postoperatorio } \\
(27)\end{array}$ & PubMed & $\begin{array}{l}\text { Cateterismo; } \\
\text { vejiga } \\
\text { urinaria }\end{array}$ & 2017 & $\begin{array}{lr}\text { En pacientes con } \\
\text { riesgo } \\
\text { complicaciones } \\
\text { postoperatorias } \\
\text { presentaron, el } \\
\text { sondaje de la vejiga } \\
\text { urinaria } \\
\text { Retencion } \\
\text { urinaria. }\end{array}$ & $\begin{array}{l}\text { Se inserta el catéter de } \\
\text { Foley en la vejiga. }\end{array}$ \\
\hline $\begin{array}{l}\text { Wahl, Graham, } \\
\text { Morris, Richman, }\end{array}$ & Pubmed & $\begin{array}{l}\text { Complicació } \\
\mathrm{n}\end{array}$ & 2018 & $\begin{array}{l}\text { Insuficiencia Renal } \\
\text { Aguda (IRA) }\end{array}$ & $\begin{array}{l}\text { No se aplicó ningún } \\
\text { rol }\end{array}$ \\
\hline
\end{tabular}




\begin{tabular}{|c|c|c|c|c|c|}
\hline $\begin{array}{l}\text { Hollis, Jones, } \\
\text { Itani, Wagner, } \\
\text { Mull, Whittle, } \\
\text { Telford, Rosen, } \\
\text { Copeland, } \\
\text { Aburns, Hawn. } \\
\text { Asociación entre } \\
\text { proteinuria } \\
\text { preoperatoria y } \\
\text { lesión renal } \\
\text { aguda } \\
\text { posoperatoria y } \\
\text { readmisión (28) }\end{array}$ & & $\begin{array}{l}\text { posoperatori } \\
\text { a renal, } \\
\text { lesión renal } \\
\text { aguda, } \\
\text { proteinuria }\end{array}$ & & & \\
\hline $\begin{array}{l}\text { Abdo, Murez, } \\
\text { Cabaniols, } \\
\text { Robert, Marchal, } \\
\text { Amadane, } \\
\text { Thezens, Iborra, } \\
\text { Thuret } \\
\text { Resultados de las } \\
\text { revisiones } \\
\text { quirúrgicas por } \\
\text { Complicaciones } \\
\text { ureterales } \\
\text { después del } \\
\text { transplante renal } \\
\text { (29) }\end{array}$ & PubMed & $\begin{array}{l}\text { Complicatio } \\
\mathrm{n} \text { ureterale; } \\
\text { fistula } \\
\text { urinaria, } \\
\text { transplante } \\
\text { de riñón }\end{array}$ & 2019 & $\begin{array}{l}\text { Estenosis uretral, } \\
\text { fuga urinaria tras el } \\
\text { trasplante renal }\end{array}$ & $\begin{array}{l}\text { No se aplicó ningún } \\
\text { rol }\end{array}$ \\
\hline $\begin{array}{l}\text { Alkandari, } \\
\text { Lieuko, Hebert, } \\
\text { Langlois, Jawa, } \\
\text { Parekh, Robison } \\
\text { Lesión renal } \\
\text { aguda en niños } \\
\text { con trasplante } \\
\text { renal (30) }\end{array}$ & PubMed & $\begin{array}{l}\text { Incidencia; } \\
\text { Pruebas; } \\
\text { renal; } \\
\text { Análisis; } \\
\text { tracto } \\
\text { urinario; } \\
\text { trasplante; } \\
\text { riñón; riesgo. }\end{array}$ & 2018 & $\begin{array}{l}\text { Infecciones del } \\
\text { tracto urinario, } \\
\text { rechazo e infección } \\
\text { del tracto urinario, } \\
\text { edad más joven, } \\
\text { niñas, injertos de } \\
\text { donante fallecidos. }\end{array}$ & $\begin{array}{l}\text { No se aplicó ningún } \\
\text { rol }\end{array}$ \\
\hline $\begin{array}{l}\text { Womble, King, } \\
\text { Hamilton, Shrout, } \\
\text { Jacobs, Duncan } \\
\text { Mayores tasas de } \\
\text { lesión renal } \\
\text { aguda } \\
\text { pacientes en } \\
\text { afroamericanos } \\
\text { con artroscopia } \\
\text { de rodilla }(31)\end{array}$ & PubMed & $\begin{array}{l}\text { Lesión renal } \\
\text { aguda; } \\
\text { profilaxis } \\
\text { antibiótica; } \\
\text { infección de } \\
\text { la } \\
\text { articulación } \\
\text { periprotésica } \\
\text {; carrera; } \\
\text { artroplastia } \\
\text { total de } \\
\text { rodilla }\end{array}$ & 2019 & Lesión renal aguda & $\begin{array}{l}\text { No se aplicó ningún } \\
\text { rol }\end{array}$ \\
\hline $\begin{array}{l}\frac{\text { Kulu, Fathi, Golri }}{\text { z, Khajeh, }} \\
\text { Sabagh, } \\
\text { Ghamarnejad, } \\
\text { Markus Mieth, } \\
\text { Ulrich, } \\
\text { Hackert, Stich, St } \\
\text { robel,Michalski, } \\
\text { Morath, Zeier, } \\
\text { Büchler, Mehrabi }\end{array}$ & PubMed & $\begin{array}{l}\text { Complicació } \\
\mathrm{n} \\
\text { hemorrágica; } \\
\text { Trasplante; } \\
\text { riñón; } \\
\text { cirujano }\end{array}$ & 2018 & $\begin{array}{l}\text { Fueron vasculares y } \\
\text { hemorrágicas, } \\
\text { Trombosis de la } \\
\text { vena renal fue la } \\
\text { complicación más } \\
\text { frecuente. }\end{array}$ & $\begin{array}{l}\text { No se aplicó ningún } \\
\text { rol de enfermería }\end{array}$ \\
\hline
\end{tabular}




\begin{tabular}{|c|c|c|c|c|c|}
\hline $\begin{array}{l}\text { Impacto de la } \\
\text { experiencia del } \\
\text { cirujano en las } \\
\text { complicaciones } \\
\text { vasculares } \\
\begin{array}{l}\text { hemorrágicas } \\
\text { posteriores }\end{array} \\
\text { trasplante al } \\
\text { riñón }(32)\end{array}$ & & & & & \\
\hline $\begin{array}{l}\text { Heung, Koyner } \\
\text { Enredo de sepsis, } \\
\text { enfermedad } \\
\text { renal crónica y } \\
\text { otras } \\
\text { comorbilidades } \\
\text { en pacientes que } \\
\text { desarrollan } \\
\text { insuficiencia } \\
\text { renal aguda (33) }\end{array}$ & PubMed & $\begin{array}{l}\text { Lesión renal } \\
\text { aguda; Cuid } \\
\text { ado } \\
\text { crítico; facto } \\
\text { res de } \\
\text { riesgo; septi } \\
\text { cemia. }\end{array}$ & 2015 & $\begin{array}{l}\text { La lesión renal } \\
\text { aguda }(L R A)\end{array}$ & $\begin{array}{l}\text { No se aplicó ningún } \\
\text { rol de enfermería }\end{array}$ \\
\hline $\begin{array}{l}\text { Adogwa, } \\
\text { Elsamadicy, } \\
\text { Sergesketter, } \\
\text { Oyeyemi, } \\
\text { Galán, Vuong, } \\
\underline{\text { Khalid, }} \text { Cheng, Bagley, } \\
\text { Karikari } \\
\text { El impacto de la } \\
\text { enfermedad } \\
\text { renal crónica en } \\
\text { los resultados } \\
\text { posoperatorios } \\
\text { en pacientes } \\
\text { sometidos a } \\
\text { descompresión y } \\
\text { fusión lumbar } \\
\text { (34) }\end{array}$ & PubMed & $\begin{array}{l}\text { Artrodesis; } \\
\text { Enfermedad } \\
\text { renal } \\
\text { crónica; } \\
\text { Complicacio } \\
\text { nes }\end{array}$ & 2018 & $\begin{array}{l}\text { Fue } \\
\text { significativamente } \\
\text { diferente entre las } \\
\text { cohortes, y el grupo } \\
\text { con Enfermedad } \\
\text { Renal crónica } \\
\text { (ERC) tuvo una } \\
\text { proporción } \\
\text { significativamente } \\
\text { mayor de pacientes } \\
\text { transferidos a la } \\
\text { unidad de cuidados } \\
\text { intensivos con } \\
\text { episodios de delirio, } \\
\text { Infección del } \\
\text { tracto urinario, } \\
\text { trombosis venosa } \\
\text { profunda. }\end{array}$ & $\begin{array}{l}\text { No se aplicó ningún } \\
\text { rol de enfermería }\end{array}$ \\
\hline $\begin{array}{l}\text { Menting, Wever, } \\
\text { Brunschot, Vliet, } \\
\text { Rovers. } \\
\text { Preacondiciona } \\
\text { miento } \\
\text { isquémico para } \\
\text { la reducción de } \\
\text { la lesión renal } \\
\text { por isquemia- } \\
\text { reperfusión }(35)\end{array}$ & $\begin{array}{l}\text { Cochrane } \\
\text { Systematic } \\
\text { Review }\end{array}$ & $\begin{array}{l}\text { Lesión renal, } \\
\text { trasplante }\end{array}$ & 2017 & $\begin{array}{l}\text { Dado a las } \\
\text { investigaciones } \\
\text { sistemáticas y de la } \\
\text { intervención } \\
\text { cardiaca realizada, } \\
\text { una de las } \\
\text { complicaciones } \\
\text { postoperarias se } \\
\text { pudo evidenciar la } \\
\text { insuficiencia renal } \\
\text { aguda. }\end{array}$ & \begin{tabular}{lrr}
\multicolumn{3}{l}{ El rol que desempeño } \\
la enfermera/o en esta \\
intervención & fue \\
asistencial ya & que \\
intervino en & la \\
realización de & la \\
diálisis de & los \\
pacientes & & \\
intervenidos y los \\
cuidados pertinentes.
\end{tabular} \\
\hline $\begin{array}{l}\text { Thompson, } \\
\text { Hosgood, } \\
\text { Nicholson, } \\
\text { Wilson. }\end{array}$ & $\begin{array}{l}\text { Cochrane } \\
\text { Systematic } \\
\text { Review }\end{array}$ & $\begin{array}{l}\text { Catéter } \\
\text { urinario, } \\
\text { complicacio } \\
\text { nes, renales }\end{array}$ & 2018 & $\begin{array}{l}\text { La extracción de un } \\
\text { stent ureteral antes } \\
\text { de la tercera semana } \\
\text { posoperatoria ( }<\text { día } \\
\text { 15) Los resultados } \\
\text { de interés fueron la }\end{array}$ & $\begin{array}{l}\text { La intervención de } \\
\text { enfermera/o, en estos } \\
\text { casos es de mantener } \\
\text { la asepsia de los } \\
\text { catéteres urinarios. }\end{array}$ \\
\hline
\end{tabular}




\begin{tabular}{|c|c|c|c|c|c|}
\hline $\begin{array}{l}\text { Extracción } \\
\text { temprana versus } \\
\text { tardía del stent } \\
\text { ureteral después } \\
\text { del trasplante de } \\
\text { riñón }(36)\end{array}$ & & & & $\begin{array}{l}\text { incidencia de } \\
\text { infección urinaria } \\
(\mathrm{IU}) \text {. }\end{array}$ & \\
\hline $\begin{array}{l}\text { Kidd, Stewart, } \\
\text { Kassis, Hom } \\
\text { Vía uretral } \\
\text { (permanente o } \\
\text { intermitente) o } \\
\text { suprapúbico } \\
\text { para la } \\
\text { cateterización de } \\
\text { corta duración } \\
\text { en adultos } \\
\text { hospitalizados } \\
\text { (37) }\end{array}$ & $\begin{array}{l}\text { Cochrane } \\
\text { Systematic } \\
\text { Review }\end{array}$ & $\begin{array}{l}\text { Cateterizació } \\
\mathrm{n} ; \text { Infección } \\
\text { Urinaria }\end{array}$ & 2015 & $\begin{array}{l}\text { Bloqueo del flujo de } \\
\text { orina. Esto causa } \\
\text { que la orina se } \\
\text { regrese y lesione } \\
\text { uno o ambos riñones } \\
\text { teniendo r como } \\
\text { resultado una } \\
\text { Infección urinaria. }\end{array}$ & $\begin{array}{l}\text { Cambio de catéter } \\
\text { uretral en los tiempos } \\
\text { indicados con la } \\
\text { finalidad de disminuir } \\
\text { la infección urinaria. }\end{array}$ \\
\hline $\begin{array}{l}\text { Roy, Fortin, Bull, } \\
\text { Doree, Trivella, } \\
\text { Hopewell } \\
\text { Intervenciones } \\
\text { para } \\
\text { nefropatía } \\
\text { crónica } \\
\text { pacientes con } \\
\text { drepanocitosis } \\
(38)\end{array}$ & $\begin{array}{l}\text { Cochrane } \\
\text { Systematic } \\
\text { Review }\end{array}$ & $\begin{array}{l}\text { Nefropatía; } \\
\text { Infección }\end{array}$ & 2017 & $\begin{array}{l}\text { Las complicaciones } \\
\text { renales que } \\
\text { producen pérdida de } \\
\text { proteína a nivel } \\
\text { renal y nefropatía } \\
\text { crónica, además de } \\
\text { Lesión } \\
\text { aguda. renal }\end{array}$ & $\begin{array}{l}\text { No se evidencio } \\
\text { intervención alguna. }\end{array}$ \\
\hline $\begin{array}{l}\text { Benítez, Noyola y } \\
\text { Victores. } \\
\text { Complicaciones } \\
\text { postoperatorias y } \\
\text { resultados } \\
\text { clínicos } \\
\text { pacientes } \\
\text { operados por } \\
\text { cáncer torácico y } \\
\text { gastrointestinal: } \\
\text { Estudio de } \\
\text { cohorte } \\
\text { prospectivo (39) }\end{array}$ & Lilacs & $\begin{array}{l}\text { Complicacio } \\
\text { nes renales; } \\
\text { cáncer } \\
\text { torácico }\end{array}$ & 2016 & $\begin{array}{l}\text { En relación a las } \\
\text { complicaciones } \\
\text { gastrointestinales, } \\
\text { se conoce a la } \\
\text { cirugía como un } \\
\text { importante factor de } \\
\text { riesgo de } \\
\text { insuficiencia renal } \\
\text { aguda. (Oliguria, } \\
\text { incremento de la } \\
\text { creatinina). } \\
\text { Porcentaje } \\
\text { complicaciones } \\
\text { postoperatoria, } \\
\text { según localización } \\
\text { de la cirugía. } \\
\text { Complicación renal } \\
\text { por cirugía } \\
\text { gastrointestinal fue } \\
(7,1 \%) \text {. } \\
\text { Complicación renal } \\
\text { por cirugía de tórax } \\
\text { fue (3\%). }\end{array}$ & $\begin{array}{lr}\text { Registro de balance } \\
\text { hídrico, resultados } \\
\text { analíticos, } \\
\text { observación } \\
\text { paciente }\end{array}$ \\
\hline $\begin{array}{lr}\text { Cunha, } & \text { Dos } \\
\text { Santos, } & \text { Costa, } \\
\text { Arimatea, Russo y } \\
\text { Oliveira. }\end{array}$ & Lilacs & $\begin{array}{l}\text { Complicacio } \\
\text { nes renales } \\
\text { postquirúrgi } \\
\text { cas }\end{array}$ & 2017 & $\begin{array}{l}\text { La infección del } \\
\text { tracto urinario } \\
\text { (ITU) representa la } \\
\text { infección } \\
\text { nosocomial más } \\
\text { común, uno de los }\end{array}$ & $\begin{array}{l}\text { Detección } \\
\text { intervención de las } \\
\text { lesiones, orientar a los } \\
\text { pacientes y sus } \\
\text { familiares, aclarando } \\
\text { dudas sobre posibles }\end{array}$ \\
\hline
\end{tabular}




\begin{tabular}{|c|c|c|c|c|c|}
\hline 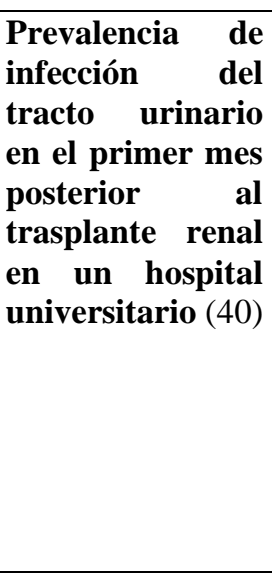 & & & & $\begin{array}{l}\text { principales sitios de } \\
\text { infección según la } \\
\text { topografía, } \\
\text { también es la } \\
\text { infección más } \\
\text { común después del } \\
\text { trasplante renal. Las } \\
\text { complicaciones más } \\
\text { frecuentes tras el } \\
\text { trasplante renal son } \\
\text { en su mayoría las } \\
\text { relacionadas con el } \\
\text { tracto urinario } \\
(28,5 \%) \text {. }\end{array}$ & $\begin{array}{l}\text { complicaciones } \\
\text { inherentes al } \\
\text { tratamiento, evitar } \\
\text { complicaciones } \\
\text { principalmente } \\
\text { infecciosas } \\
\text { estrategias o } \\
\text { combatirlas, medidas } \\
\text { de higiene de manos y } \\
\text { la adecuada } \\
\text { manipulación del } \\
\text { catéter urinario. }\end{array}$ \\
\hline $\begin{array}{l}\text { Pereira, Badaoui, } \\
\text { Dantas, Sanches, } \\
\text { Alvares, } \\
\text { Scaranello, } \\
\text { Castro, Silva y } \\
\text { Laranja } \\
\text { Lesión renal } \\
\text { aguda en el } \\
\text { postoperatorio } \\
\text { de cirugías no } \\
\text { cardíaca } \\
\text { pacientes en } \\
\text { recuperación en } \\
\text { la unidad de } \\
\text { cuidados } \\
\text { intensivos (41) }\end{array}$ & Lilacs & $\begin{array}{l}\text { Complicacio } \\
\text { nes renales } \\
\text { postquirúrgi } \\
\text { cas }\end{array}$ & 2016 & 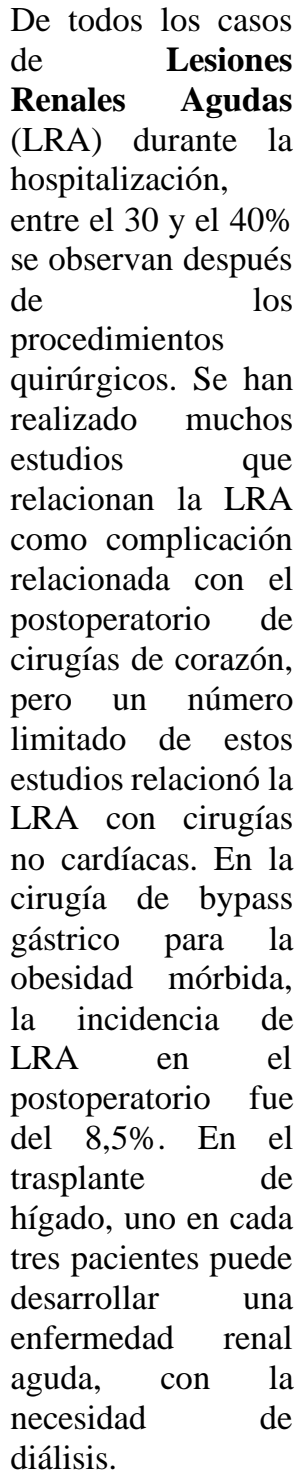 & $\begin{array}{l}\text { En este artículo no se } \\
\text { evidencio } \\
\text { intervención } \\
\text { enfermería. }\end{array}$ \\
\hline $\begin{array}{l}\text { Hernández; } \\
\text { González. } \\
\text { Complicaciones } \\
\text { anestésicas } \\
\text { intraoperatorias } \\
\text { y posoperatorias } \\
\text { de la } \\
\text { nefrolitotomía }\end{array}$ & Lilacs & $\begin{array}{l}\text { Complicacio } \\
\text { nes Renales } \\
\text { posoperatori } \\
\text { a. }\end{array}$ & 2018 & $\begin{array}{l}\text { La complicación } \\
\text { más relevante } \\
\text { Insuficiencia renal } \\
\text { aguda y sépticas. }\end{array}$ & $\begin{array}{l}\text { No se aplicó ningún } \\
\text { rol de enfermería en el } \\
\text { presente artículo. }\end{array}$ \\
\hline
\end{tabular}




\begin{tabular}{|l|l|l|l|l|}
\hline $\begin{array}{l}\text { percutánea en } \\
\text { pacientes con } \\
\text { litiasis } \\
\text { coraliforme (42) }\end{array}$ & & & & \\
\hline $\begin{array}{l}\text { García, Chaparro, Lilacs } \\
\text { Hoz. }\end{array}$ & $\begin{array}{l}\text { Complicacio } \\
\text { nes Renales } \\
\text { posoperatori } \\
\text { a. }\end{array}$ & $\begin{array}{l}\text { Cólico } \\
\text { renoureteral y } \\
\text { hematuria asociado } \\
\text { a la presencia del } \\
\text { catéter doble. } \\
\text { percutitotomía } \\
\text { ambulatoria: } \\
\text { serie de casos y } \\
\text { revisión de la } \\
\text { literatura (43) }\end{array}$ & $\begin{array}{l}\text { No se aplicó ningún } \\
\text { rol de enfermería en el } \\
\text { presente artículo. }\end{array}$ \\
\hline
\end{tabular}

\section{Análisis de los resultados}

En los resultados de esta revisión bibliográfica se pudo comprobar que, de los 19 artículos tomados para revisión bibliográfica solo se evidenciaron en 6 artículos el rol asistencial que desempeña la enfermera/o ante las complicaciones postquirúrgicas renales y de las vías urinarias.

Por otra parte mediante el análisis correspondiente se evidencio que las complicaciones postquirúrgicas renales y de las vías urinarias abarcan un factor de complejidad tras las intervenciones quirúrgicas, por una parte encontramos la Insuficiencia Renal Aguda (IRA) siendo la más prevalente es decir ocupando el primer lugar, así lo manifestaron Menting, Wever, Brunschot, Vliet y Rovers (35) con un $72 \%$ de los resultados, en segundo lugar encontramos la Infeccion del Tracto Urinario (ITU) lo señalaron Adogwa, Elsamadicy, Sergesketter, Oyeyemi, Galán ,Vuong, Khalid, Cheng, Bagley y Karikari (34) con un $52.9 \%$.

En tercer lugar encontramos a la Retencion Urinaria (RU) quien menciono Krajka y Dorobek (27) con un $23 \%$ de las personas que padecieron dicha complicación, en cuarto lugar encontramos la Estenosis uretral; según sus estudios realizados por Abdo, Murez, Cabaniols, Robert, Marchal, Amadane, Thezens, Iborra y Thuret (29) con un $5.8 \%$, en quinto lugar encontramos la Trombosis de la vena renal así lo dio a conocer Kulu, Fathi, Golriz,
Khajeh, Sabagh, Ghamarnejad, Markus Mieth, Ulrich, Hackert, Stich, Strobel,Michalski, Morath, Zeier, Büchler y Mehrabi (32) con un $2.6 \%$ y por ultimo ocupando el sexto lugar encontramos el Cólico renoureteral y hematuria con un $2 \%$ así lo señaló García, Chaparro y Hoz (43).

Todos estos datos relevantes han sido tomados mediante las revisiones bibliográficas señaladas anteriormente.

\section{Conclusiones}

- La mayoría de los artículos tomados como estudio, sobre la caracterización del rol de la enfermera ante las complicaciones postquirúrgicas renales y de las vías urinarias no está evidenciada, solo 6 de ellos demostró como resultado el desempeño oportuno ante estas complicaciones postquirúrgicas lo cual fue el rol asistencial.

- Entre las complicaciones postquirúrgicas encontradas en las revisiones bibliográficas se pudo conocer que en primer lugar se encuentra la Infección Renal Aguda, siguiendo la Infección del tracto urinario, la Retención Urinaria, seguido de la Estenosis uretral, Trombosis de la vena renal y por último sin dejar a un lado el cólico renoureteral y hematuria.

\section{Colaboradores en la Investigación:}

Alcívar Quimis Edison Eduardo; Guamán Guanca José Humberto; Moran Pibaque Jamilex Anabel; Ortiz Hernández Diana Estefanía; Ruiz Sánchez Sasha Yamiledt; Santana Tigua Jonathan David; Solís Gutiérrez John Alex; Tóala Suarez Ginger Giselle; Velíz Piloso Evelin Jamileth; Villacreses Arteaga Kerly Margarita.

Carrera de Enfermería de la Universidad Estatal del Sur de Manabí. 


\section{Bibliografía}

1. Oneys del Carmen De Arco- Canoles, Zuleima Karina Suarez-Calle. Rol de los profesionales de enfermría en el sistema de salud Colombiano. 2018 Abril 13.

2. Maricela Torres Esperón, Caridad Dandicourt Thomas, Aida Rodríguez Cabrera. Funciones o Roles de la Enfermera. Yo amo Enfermería. 2029 Mayo 21.

3. Cátedra de cirugía. Postoperatorio. Universidad Nacional de Córdova. 2011 Agosto 8.

4. Silvio R Zúñiga, José Gómez Marquez. Complicaciones Post-Operatorias Abdominales. Bsv. 1974; 42.

5. Complicaciones Renales. Bd. 2016 Junio.

6. María C Sánchez. Universidad del Azuay. [Online].; 2014 [cited 2020 Julio 10. Available from: http://dspace.uazuay.edu.ec/bitstream/datos/3940/1/10544.pdf.

7. Laura Estefania Alvarado Robles, Dayra Gisella Angulo DE León. IMPACTO DEL ROL DE ENFERMERIA EN PACIENTES CON INSUFICIENCIA RENAL CRÓNICA. Tesis de grado previo a la obtención del titúlo de Enfermera. Guayaquil:; 2013-2014.

8. Laura Estefania Alvarado Robles, Dayra Gisella Angulo de León. Impacto del Rol de enfermeria en pacientes con complicaciones renales. Tesis de Licenciatura en Enfermería. Guayaquil: Universidad de Guayaquil; 2014-2015.

9. Maricela Torres Esperón, Caridad Dandicourt Thomas y Aida Rodríguez Cabrera. Funciones de enfermería en la atención primaria de salud. Scielo. 2005 Mayo - Agosto; 21(3-4).

10. Bertha S, Rodríguez A. Características del PAE. In ; 2015.

11. Universidad internacional de Valencia. Proceso de atención de enfermería: concepto y etapas. VIU. 2018 Marzo 21.

12. Zahira Ángel, Esperanza Ángel. Cuidados de enfermería en el paciente con enfermedad renal crónica. Articulo de revision. Colombia: Fundación Universitaria de Ciencias de la Salud; 2016 Julio 19.

13. Priscila Gonzáles Salcedo; Alida Mireya Chaves Reyes. Proceso de atención de enfermería desde la perspectiva docente. Redalyc. 2009 Julio; 11(2).

14. Nahum Montahud Rubio. Sistema excretor: características, partes y funcionamiento. Psicología y mente. 2017 Marzo.

15. National Institute of Diabetes and Digestive and Kidney Diseases. Los riñones y su funcionamiento. NIH. 2018 Junio.

16. Healthwise. Filtración glomerular (GFR). Cigna. 2019 Agoato 11.

17. Julia Máxima Uriarte. Sistema excretor. Características.com. 2019 Diciembre.

18. Asociación Española Contra el Cancer. La Vejiga Anatomía. AECC. 2018.

19. ELSEVIER. Incidencia de la infección urinaria en pacientes postoperados de cirugía cardiaca: estudio comparativo según el dispositivo de sondaje. ELSEVIER. 2015 Abril-Junio; 26(2).

20. Mayo Clinic. Infección de las vías urinarias. Infección de las vías urinarias. 2019 Marzo 19. 
21. Universidad Peruana Cayetano Heredia. Frecuencia de retención urinaria como complicación post operatoria de cirugías de prolapso de órganos pélvicos en el Hospital Nacional Arzobispo Loayza. 2017 - 2019 Enero- Diciembre.

22. Manual MSD. Lesiones ureterales. MSD. 2016 Agosto.

23. Manual MSD. Traumatismos vesicales. MSD. 2015 Febrero.

24. Elsa Solá, Pere Ginés. Síndrome Hepatorrenal. Sociedad Española de Nefrología. 2018 Abril 27.

25. B Bzoma, J Kostro, Un Hellmann, A Chamienia, S Hać, A Dębska-Ślizień, Z Śledziński. PudMed. [Online].; 2018 [cited 2020 Agosto 10. Available from: https://pubmed.ncbi.nlm.nih.gov/30056916/.

26. Steven J. Skube, Stephen A. Katz, Jeffrey G Chipman, Christopher J Tignanelli. PubMed. [Online]; 2018 [cited 2020 Agosto 10. Available from: https://pubmed.ncbi.nlm.nih.gov/29304308/.

27. Emilia Pawłowska-Krajka, Adam Dorobek. PudMed. [Online].; 2017 [cited 2020 Agosto 10. Available from: https://pubmed.ncbi.nlm.nih.gov/29410894/.

28. Tyler S Wahl, Laura A Graham, Melanie S Morris, Joshua S Richman, Robert H Hollis, Caroline E Jones, Kamal M Itani, Todd H. Wagner, Hillary J Mull, Jeffrey C Whittle, Gordon L. Telford, Am K Rosen, Laurel A Copeland, Edith Aburns, Mary T Hawn. PubMed. [Online].; 2018 [cited 2020 Agosto 11. Available from: https://pubmed.ncbi.nlm.nih.gov/29971429/.

29. N Abdo, T Murez, L Cabaniols, M Robert, S Marchal, N Amadane, S Thezens, F Iborra, R Thuret. PubMed. [Online].; 2019 [cited 2020 Agosto 11. Available from: https://pubmed.ncbi.nlm.nih.gov/31400962/.

30. Omar Alkandari, Lieuko, Diane Hebert, Valerie Langlois, Natasha A Jawa, Rulan S Parekh, Lisa A Robison. PubMed. [Online].; 2018 [cited 2020 Agosto 11. Available from: https://pubmed.ncbi.nlm.nih.gov/30242029/.

31. Tanner N Womble, John D King, Dustin H Hamilton, Max A Shrout, Cale A Jacobs, Stephen T. Duncan. PubMed. [Online].; 2019 [cited 2020 Agosto 11. Available from: https://pubmed.ncbi.nlm.nih.gov/30824293/.

32. Yakup Kulu, Parham Fathi, Mohammad Golriz, Elias Khajeh, Mohammadsadegh Sabagh, Omid Ghamarnejad, Markus Mieth, Alexis Ulrich, Thilo Hackert, Beat P Müller-Stich, Oliver Strobel, Christoph Michalski, Christian Morath, Martin Zeie. PubMed. [Online].; 2018 [cited 2020 Agosto 12. Available from: https://pubmed.ncbi.nlm.nih.gov/30236441/.

33. Michael Heung, Jay L Koyner. PubMed. [Online].; 2015 [cited 2020 Agosto 12. Available from: https://pubmed.ncbi.nlm.nih.gov/25795497/.

34. Owoicho Adogwa, Aladine A Elsamadicy, Amanda Sergesketter, Deborah Oyeyemi, Diego Galan, Victoria D Vuong, Syed Khalid, Joseph Cheng, Carlos A Bagley, Isaac O Karikari. PubMed [Online].; 2017 [cited 2020 Agosto 15. Available from: https://pubmed.ncbi.nlm.nih.gov/29109065/.

35. Theo P Menting, Kimberley E Wever, Denise MD Ozdemir-van Brunschot, Daan JA Van der Vliet, Maroeska M Rovers, Michiel C Warle. Cochrane Library Riviews. [Online].; 2017 [cited 2020 Agosto $15 . \quad$ Available from: https://www.cochranelibrary.com/cdsr/doi/10.1002/14651858.CD010777.pub2/full/es?highlightA bstract=renal\% 7Ccomplicaciones $\% 7$ postoperatorias \% 7Cpostoperatoria\% 7Ccomplicacion $\% 7 \mathrm{Cp}$ ostoperatori\% 7 Crenales.

36. Emily R ThompsonSarah A HosgoodMichael L NicholsonColin H Wilson. Cochrane Library Reviews. [Online]; 2018 [cited 2020 Agosto 15. Available from: https://www.cochranelibrary.com/cdsr/doi/10.1002/14651858.CD011455.pub2/full/es?highlight 
Abstract=renal\% 7Ccomplicaciones\%7Cpostoperatorias\%7Cpostoperatoria\%7Ccomplicacion\% 7Cpostoperatori\%7Crenales.

37. Emily A Kidd; Fiona Stewart; Nadine C Kassis; Emily Hom. Cochrane Systematic. [Online].; 2015 [cited $2020 \quad$ Agosto $17 . \quad$ Available from: https://www.cochranelibrary.com/cdsr/doi/10.1002/14651858.CD004203.pub3/full/es?highlightA bstract=vias\%7Curetral\% 7Cintermitent\%7Cpermanent\%7Curetrales\%7Cintermitentes\%7Cperma nentes\%7Ci\%7Co.

38. Noemi BA Roy; Patricia M Fortin; Katherine R Bull; Carolyn Doree; Marialena Trivella; Sally Hopewell. Cochrane Systematic Review. [Online].; 2017 [cited 2020 Agosto 15. Available from: https://www.cochranelibrary.com/cdsr/doi/10.1002/14651858.CD012380.pub2/full/es?highlightA bstract=con $\% 7 \mathrm{Cdisminuir} \% 7 \mathrm{Cen} \% 7 \mathrm{Cparas} \% 7 \mathrm{Cintervencion} \% 7 \mathrm{Crenales} \% 7 \mathrm{Co} \% 7 \mathrm{Crenal} \% 7 \mathrm{Cpac}$ ient \% 7Ccomplicaciones\%7Cprevenir\%7Cpara\%7Cdrepanocitosi\%7Cdrepanocitosis\%7Cpaciente s\% 7.

39. Benitez, Noyola y Victores. Complicaciones postoperatorias y resultados clínicos en pacientes operados por cáncer torácico y gastrointestinal: Estudio de cohorte prospectivo. Revista Brasileira de Terapia Intensiva. 2016 Marzo; 28(1).

40. Cunha, Dos Santos , Costa, Arimatea, Russo y Oliveira. Prevalencia de infección del tracto urinario en el primer mes posterior al trasplante renal en un hospital universitario. Revista de enfermagem Uerj Nursing Journal. 2017 Abril; 25.

41. Pereira, Badaoui, Dantas, Sanches, Alvares, Scaranello, Castro, Silva y Laranja. Lesão renal aguda no pós-operatório de cirurgias não cardíacas em pacientes com recuperação na unidade de terapia intensiva. Revista Sociedade Brasileira de Clínica Médica. 2016; 14(4).

42. González, Hernández. Complicaciones anestésicas intraoperatorias y posoperatorias de la nefrolitotomía percutánea en pacientes con litiasis coraliforme. scielo. 2018 enero-abril.

43. Carlos Eduardo Hernández García, Diana Chaparro. Gustavo Ramos,José de la Hoz. Nefrolitotomía percutánea ambulatoria: serie. Urol. colomb. 2018.

44. Alondra Reyes. 2017 Marzo 29.

45. Mariano A. Núñez, Bernardo Chiconi, Gonzalo Linares, Federico Aveni, Mauricio Pilot, Germán Albino. Litiasis renal: ¿Puede el abordaje percutáneo resolver todos los casos? Rev. argent. urol. (1990). 2018. 